\title{
Reversing a granular flow on a vibratory conveyor
}

\author{
R. Grochowski and P. Walzel \\ Mechanische Verfahrenstechnik, Universität Dortmund, D-44227 Dortmund, Germany \\ M. Rouijaa, C.A. Kruelle*, and I. Rehberg \\ Experimentalphysik V, Universität Bayreuth, D-95440 Bayreuth, Germany
}

(Dated: November 25, 2003)

\begin{abstract}
Experimental results are presented on the transport properties of granular materials on a vibratory conveyor. For circular oscillations of the shaking trough a non-monotonous dependence of the transport velocity on the normalized acceleration $\Gamma$ is observed. Two maxima are separated by a regime, where the granular flow is much slower and, in a certain driving range, even reverses its direction. A similar behavior is found for a single solid body with a low coefficient of restitution, whereas an individual glass bead of $1 \mathrm{~mm}$ diameter is propagated in the same direction for all accelerations.
\end{abstract}

PACS numbers: 45.70.Mg 45.50.-j 05.60.Cd

The controlled transport of bulk cargoes by means of vibratory conveyors is of major importance for a whole variety of industrial processes [1, 2, 3]. The granular material is usually (i) agitated by a stick-slip drag on a horizontally vibrated deck with asymmetric forward and backward motions, (ii) forced to perform ballistic flights if the vertical component of the acceleration exceeds gravity, or (iii) can be transported horizontally by a vertically oscillating asymmetric sawtooth-shaped profile of the base 4,5$]$.

Since these transport phenomena involve the nonlinear interaction of many-particle systems with complex behavior leading to self-organized spatiotemporal patterns, the investigation of vibrating granular materials has become a challenging subject to physicists, too 6, 7]. Such intriguing phenomena as surface waves 8 , 9, 10, 11], organized clusters [12] or segregation effects [13, 14, 15] have attracted a lot of attention.

In this letter, we introduce a conveyor system with convertible modes of oscillation based on the combined forces of four rotating unbalanced masses. First results are reported on the surprising transport properties caused by circular vibrations of the trough.

The circular vibratory conveyor, shown schematically in Fig. 1(a), is a prototype apparatus specially developed to investigate the transport under principal oscillation modes, i.e. linear, elliptical, and circular for a long running time, without disturbing boundary conditions. In our experiments, the transporting trough has the form of a horizontally oriented ring with radius $R$ $=22.5 \mathrm{~cm}$ and width $w=5 \mathrm{~cm}$ and is suspended on adjustable columns via elastic bands. By means of four driving units, symmetrically positioned below the trough, the conveyor can vibrate with defined amplitude and oscillation pattern along its entire circumference. This motion can be described by a trajectory performed on

*Electronic mail: christof.kruelle@uni-bayreuth.de

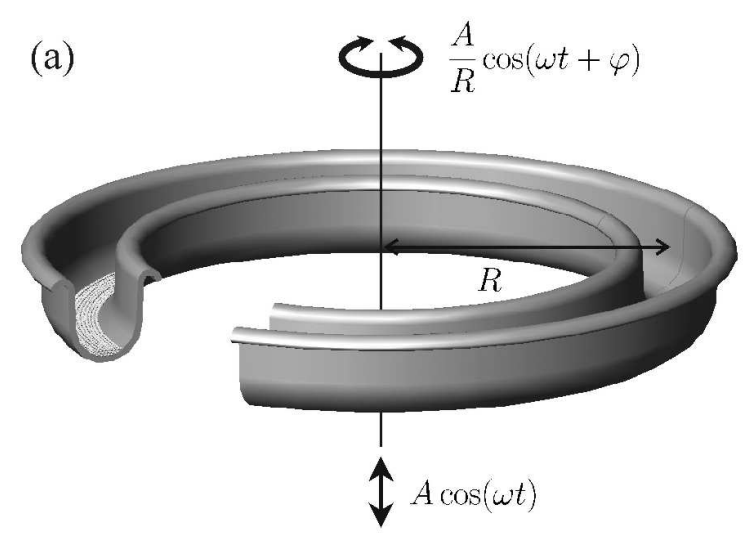

(b)

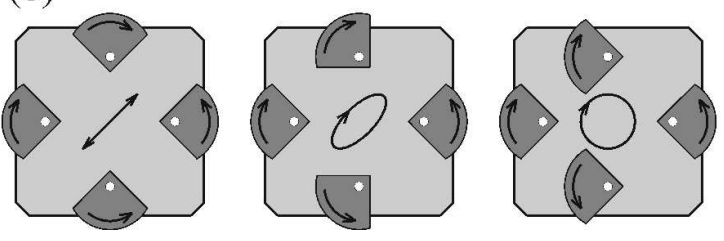

FIG. 1: (a) Sketch of the circular vibratory conveyor. By adjusting the phase shift $\varphi$ between the vertical and the torsional vibration each part of the annular trough can be forced on a specified trajectory. (b) Schematic side views of a driving unit with four rotating unbalanced masses, for three principal modes of oscillation: linear $(\varphi=0)$, elliptical $\left(\varphi=\frac{\pi}{4}\right)$, and $\operatorname{circular}\left(\varphi=\frac{\pi}{2}\right)$.

a cylindrical surface, consisting of a vertical oscillation $z(t)=A \cos (\omega t)$ superposed with a torsional vibration $\phi(t)=A / R \cos (\omega t+\varphi)$ around the symmetry axis of the apparatus, where $\varphi$ is the fixed phase shift between the two oscillations. Note that, since the ratio $A / R$ is only about 1 percent, the path of each segment of the tray can be considered to lie almost in a vertical plane. If, for example, the phase shift $\varphi$ is chosen to be $\pi / 2$, then each point on the trough traces a circular path in a vertical plane tangent to the trough at that point. 


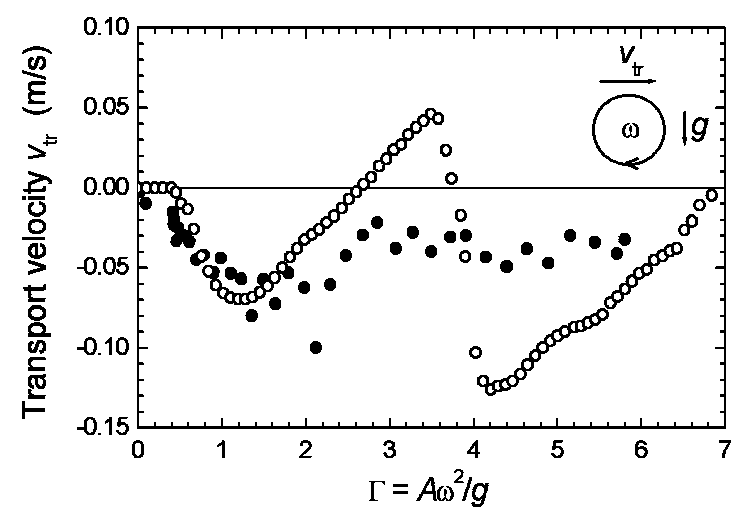

FIG. 2: Transport velocity $v_{\text {tr }}(\Gamma)$ of a granular flow $(\approx 300000$ glass beads with $1 \mathrm{~mm}$ diameter, $\circ)$ on the vibratory conveyor, compared with the mean velocity of one single glass bead $(\bullet)$. Conveyor amplitude $A_{\infty}=1.7 \mathrm{~mm}$.

For achieving this kind of motion with the inherent possibility to generate different modes of oscillation a special adjustable drive is acquired. Unbalanced-mass vibrators are well established in industrial applications since long time. Their working principle is based on a centrifugal force $F=m_{\mathrm{u}} r_{\mathrm{u}}(2 \pi f)^{2}$ produced by an unbalanced mass $m_{\mathrm{u}}$ rotating with frequency $f$, with $r_{\mathrm{u}}$ being the distance between the center of gravity of the unbalanced (eccentric) mass and its rotation axis. A motor driving a load $M$ with one single unbalanced mass will create, for frequencies well above resonance, a circular vibration with an amplitude $A_{\infty}=r_{\mathrm{u}} m_{\mathrm{u}} / M$. A linear motion can be excited by the joint action of two equal vibrators rotating in opposite directions. In consequence, by combining two such linear vibrators oriented perpendicularly to each other, it is possible to generate any desired Lissajous figure by adjusting the phase shift $\varphi$ between the two oscillations. Three examples of possible modes are shown in Fig. 1(b).

Each driving unit is built as the above described system of four unbalanced masses, which are placed vertically on both sides of the unit. The vibrators are fixed to the carrying plates by bolts located in circular grooves, which enable the adjustment of the vibration angle $\alpha$ between $8^{\circ}$ and $82^{\circ}$. The oscillation amplitude can be adjusted in steps by changing the number of impaled unbalanced masses.

The four driving units are coupled to the motor via a common central gear box, which keeps all drives in the same phase. The driving torque is transmitted from the gear box to the vibrators by use of rotating rods connected with compensation clutches. The conveyor is driven by an electric motor (Siemens Combimaster 1UA7, with integrated frequency inverter). By changing its rotation frequency $f$, the number of unbalanced masses $m_{\mathrm{u}}$, and their angular alignment, the adjustment of all required oscillation conditions is possible.

The circular vibratory conveyor has been designed as

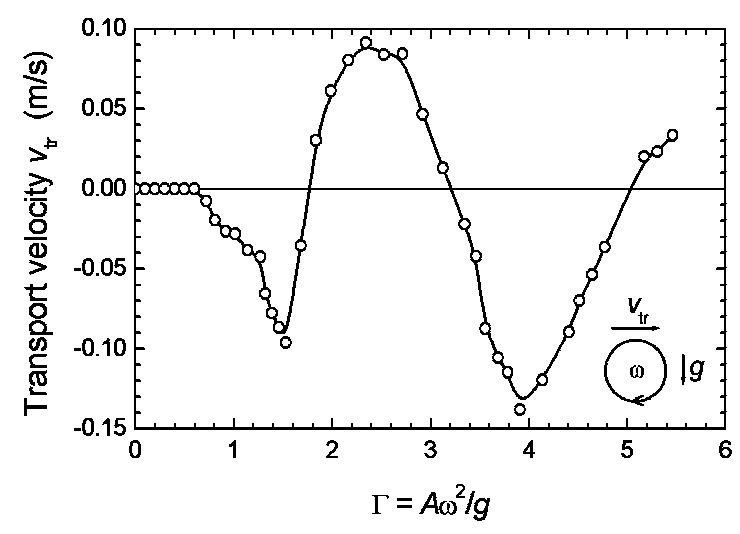

FIG. 3: Transport velocity $v_{\mathrm{tr}}(\Gamma)$ of an object with low coefficient of restitution (sand-filled 'fingertip'). Conveyor amplitude $A_{\infty}=1.7 \mathrm{~mm}$.

an 'over-resonance' apparatus. Low spring constants of the suspensions set the resonance frequency to $f_{0} \approx 4.5$ $\mathrm{Hz}$. For frequencies $f>15 \mathrm{~Hz}$ the change of the amplitude is less than $10 \%$. Thus the system can be assumed as working with constant amplitude independent from the frequency. However, for execution of accurate experiments the frequency response of the amplitude

$$
A(f)=A_{\infty} \frac{f^{2}}{\sqrt{\left(f_{0}^{2}-f^{2}\right)^{2}+\left(2 \zeta f_{0} f\right)^{2}}}
$$

(with damping $\zeta=0.08 \pm 0.01$ ) has been measured. The dimensionless acceleration of the conveyor $\Gamma=A(f)$. $(2 \pi f)^{2} / g$, where $g$ is the gravitational acceleration, can be varied by changing the rotation frequency $f$ of the unbalanced masses in the range $0<\Gamma<7$.

For the case of circular vibrations we define the transport direction of the granulate positive ('forward'), if the rotational direction of the vibration - seen from outside the apparatus - is clockwise while the particles move to the right, or vice versa. To be more specific, we assign to the circular motion an axial vector $\vec{\omega}$ (see inset of Fig. 2 ). A positive transport velocity $\vec{v}_{\mathrm{tr}}$ is found, whenever the product $\vec{v}_{\mathrm{tr}} \cdot(\vec{g} \times \vec{\omega})$ is positive.

The granulate used consists of $\approx 300000$ glass beads with diameter $d=1 \mathrm{~mm}$, yielding a layer height of $\approx 5 d$. The velocity $v_{\mathrm{tr}}$ of the granular flow was measured as a function of the dimensionless acceleration $\Gamma$ in the range between 0 and 7 . The non-monotonous dependence $v_{\text {tr }}(\Gamma)$ is shown in Fig. 2. Below a critical value $\Gamma_{c} \approx 0.45$ the grains stay at rest, i.e., they follow the agitation of the tray without being transported. The onset of particle motion is restrained by frictional forces between grains and the substrate. For accelerations above this threshold the granular material becomes fluidized. Individual particles are unblocked and begin to move freely on top of each other. A net granular flow with constant velocity is observed. Note that this behavior is found already in a regime $\Gamma<1$ with a vertical acceleration less than gravity. In order to be transported, the granular material 


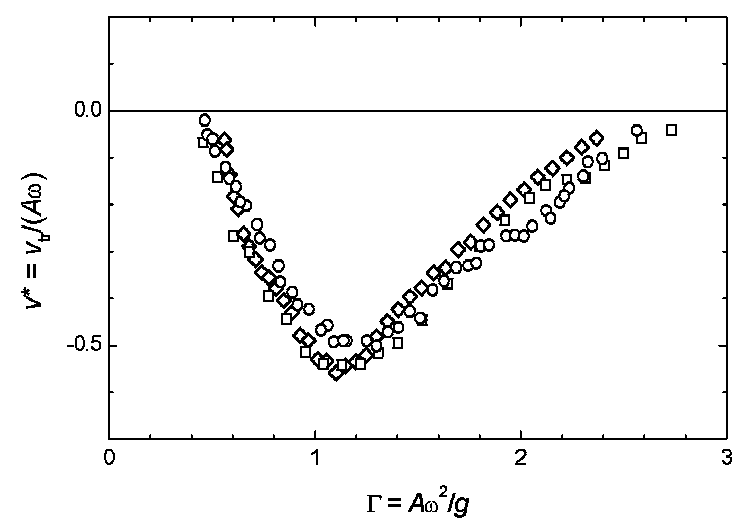

FIG. 4: Scaled velocity $v^{*}=v_{\text {tr }} /(A \omega)$ of the granular flow. The experimental data were obtained for three different conveyor amplitudes: $A_{\infty}=1.0 \mathrm{~mm}$ (०), $A_{\infty}=1.7 \mathrm{~mm}(\square)$, and $A_{\infty}=2.5 \mathrm{~mm}(\diamond)$, respectively.

does not have to leave the ground. It is sufficient to overcome the frictional forces at the bottom of the granular layer. This transport mechanism due to stick-slip drag on a horizontally vibrated deck with asymmetric forward and backward motions is well known to engineers as 'sliding'.

If $\Gamma$ exceeds 1 , the vertical component of the circular acceleration will cause the grains to detach from the bulk followed by a flight on a ballistic parabola. The corresponding transport mechanism is called 'throwing'.

In our experiments the transport velocity has a first maximum at $\Gamma=1.2$. A second maximum is observed at $\Gamma=4.2$. In between, the granular flow is slower and even reverses its direction for $2.6<\Gamma<3.8$.

In contrast, a single $1 \mathrm{~mm}$ glass bead is propagated on this vibratory conveyor in the same direction for all accelerations. The high coefficient of restitution $\varepsilon \approx 0.9$ for collisions between the glass bead and the carbon-fiber tray leads, especially for high $\Gamma$-values, to an almost 'random walk' of the particle in the conveyor with a slight tendency to propagate in one direction. This is reflected in the large scatter of the net transport velocity.

In order to study the transport mechanism for a single body in a more controlled fashion ('sandbag test' 16]) we designed an object with a very low coefficient of restitution and non-spherical shape. The fingertip of a rubber glove was filled with sand. This single object shows qualitatively the same behavior as the granulate (see Fig. 3). The regime of velocity reversal is, however, shifted to lower values $(1.8<\Gamma<3.2)$.

By varying the unbalanced mass, the amplitude $A_{\infty}$ of the circular vibration has been changed between 1.0 and $2.5 \mathrm{~mm}$. We find that the transport velocity increases linearly with the amplitude. In order to obtain a dimensionless graph, the granular velocity is scaled by the intrinsic speed of the driving apparatus, i.e. the circular velocity $A \omega$ of the tray. The resulting master curve (Fig. 4) shows clearly that the onset of particle transport

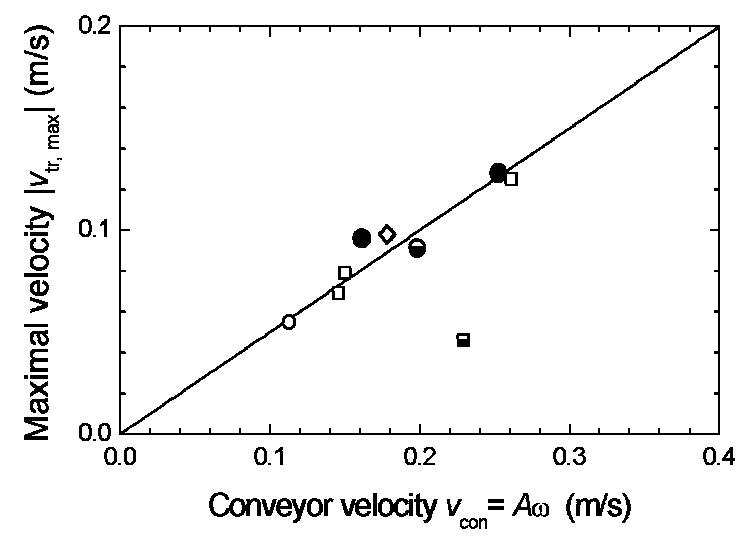

FIG. 5: Maximal velocity of the granular flow and the fingertip (-) on the vibratory conveyor. The solid line indicates $v=0.5 \cdot A \omega$, i.e. one half of the local conveyor velocity $v_{\text {con }}$. The experimental data were obtained for three different conveyor amplitudes: $A_{\infty}=1.0 \mathrm{~mm}(\circ), A_{\infty}=1.7 \mathrm{~mm}(\square)$, and $A_{\infty}=2.5 \mathrm{~mm}(\diamond)$, respectively. Half-filled symbols indicate reversed flow.

depends only on $\Gamma$. This supports the notion that the particles have to overcome a (frictional) force.

An alternative approach would have been to scale the vibratory agitation with the Froude number $\mathrm{Fr}=$ $(A \omega)^{2} / g d=\Gamma A / d$, i.e. a typical dimensionless kinetic energy. However, the corresponding plot fails to produce a similar data collapse.

Independent on the oscillation amplitude $A$ we observe that the maximal achievable transport velocity is equal to $0.5 \cdot A \omega$ (Fig. 5). For the single particle with low coefficient of restitution this is true for both directions of motion, even at higher accelerations. The reversed granular flow, however, does not attain this limit.

In conclusion, the vibratory conveyor system introduced here opens up the possibility to investigate the transport properties of granular materials in a systematic way. Our results show that under certain conditions not even the direction of the granular flow can be predicted a priori. The delicate interactions of the particles with the support as well as among themselves have to be taken into account. First clues indicate that a detailed analysis of the frictional forces becomes important. A simple model [1] for a frictional block on a horizontally oscillating bed has been introduced recently, which provides a relaxational mechanism between static and dynamic friction. This suggests that before tackling the full problem of many interacting particles it is even rewarding to investigate the complex behavior of a single object under the influence of a controlled environment.

We would like to thank H. Elhor, S.J. Linz, F. Landwehr, S. Strugholtz, and T. Schnautz for valuable discussions. This work was supported by the Deutsche Forschungsgemeinschaft (DFG-Sonderprogramm 'Verhalten granularer Medien'). 
[1] G. Pajer, H. Kuhnt, and F. Kuhnt, Fördertechnik Stetigförderer (VEB Verlag Technik, Berlin, 5th edn., 1988), pp. 226-255.

[2] F.J.C. Rademacher and L. Ter Borg, Eng. Res. 60, 261 (1994).

[3] E.M. Sloot and N.P. Kruyt, Powder Technol. 87, 203 (1996).

[4] I. Derényi, P. Tegzes, and T. Vicsek, Chaos 8, 657 (1998).

[5] Z. Farkas, P. Tegzes, A. Vukics, and T. Vicsek, Phys. Rev. E 60, 7022 (1999).

[6] H.M. Jaeger, S.R. Nagel, and R.P. Behringer, Rev. Mod. Phys. 68, 1259 ( 1996).

[7] L.P. Kadanoff, Rev. Mod. Phys. 71, 435 (1999).

[8] S. Douady, S. Fauve, and C. Laroche, Europhys. Lett. 8, 621 (1989).

[9] H.K. Pak and R.P. Behringer, Phys. Rev. Lett. 71, 1832 (1993).
[10] F. Melo, P. Umbanhowar, and H.L. Swinney, Phys. Rev. Lett. 72, 172 (1994).

[11] T. Metcalf, J.B. Knight, and H.M. Jaeger, Physica A 236, 202 (1997).

[12] G. Strassburger and I. Rehberg, Phys. Rev. E 62, 2517 (2000).

[13] S. Aumaître, C.A. Kruelle, and I. Rehberg, Phys. Rev. E 64, 041305 (2001).

[14] S. Aumaître, T. Schnautz, C.A. Kruelle, and I. Rehberg, Phys. Rev. Lett. 90, 114302 (2003).

[15] S.J. Moon, D.I. Goldman, J.B. Swift, and H.L. Swinney, Phys. Rev. Lett. 91, 134301 (2003).

[16] F.J.C. Rademacher, Bulk Solids Handling 15, 41 (1995).

[17] G. Metcalfe, S.G.K. Tennakoon, L. Kondic, D.G. Schaeffer, and R.P. Behringer, Phys. Rev. E 65, 031302 (2002). 